\title{
The opportunity for apps
}

\author{
The advent of smart phones and tablet devices should give scientists exciting new opportunities for \\ collecting, managing and interfacing with data.
}

One only has to take a quick glance around cafés, trains and offices to realize the rising popularity of smart phones and tablets. Indeed, Apple's iPhone and iPad, which run an operating system called iOS, as well as smart phones running Google's Android operating system, are fast becoming a 'must have' accessory for those that desire convenient access to the Internet while travelling. A major selling point of these devices is the ease at which new software - known as 'apps' - can be downloaded from the Apple iTunes store or the Android Market for immediate use. Apps cover an incredible diversity of functions, ranging from games, personal organizers and productivity aides to tools for conveniently managing photos, music and e-books. However, so far very few apps have been built to serve the needs of scientists. Arguably, the most successful and useful app so far is Papers for iOS (www.mekentosj.com/papers), which provides scientists with a new, convenient way of managing, organizing and storing research papers in a personal and portable library. In addition, several journal publishers such as the Institute of Physics, Nature Publishing Group (NPG) and the American Association for the Advancement of Science have recently released apps that allow their content to be easily viewed and managed by mobile devices. However, that is about as far as scientific apps have progressed.

One firm that's trying to change the situation is Digital Science (www.digitalscience.com), a sister company of NPG that was launched in December 2010. Timo Hannay, managing director of Digital Science, says the firm is busy developing the innovative digital tools that scientists will need in the future to manage, visualize, interact with and share data in more convenient way.

"We're a new division of Macmillan with a mission to support the needs of scientists and researchers. NPG does this by providing the high-quality content they need, whereas Digital Science is about providing the technology they need," explained Hannay.

"When it comes to data management, scientists have better tools for organizing their personal music and photos than the data collected in their lab."

One of the possibilities that Hannay finds most exciting is the opportunity for tablet devices to create a true end-to-end digital workflow for scientists. "We're seeing an explosion in the number of tablet devices, and they have huge potential for use in the lab," commented Hannay. "If you think about it, the only thing that isn't digital in the workflow for data is a scientist's lab notebook, and the tablet presents an opportunity to change this."

Beyond data management, the other big scientific opportunity for tablet and mobile devices could be for providing convenient control over apparatus in the lab. As described in detail on page 255 of this issue, researchers from the Universities of Glasgow and Bristol in the UK have now developed an app that allows scientists to wirelessly control optical tweezers and

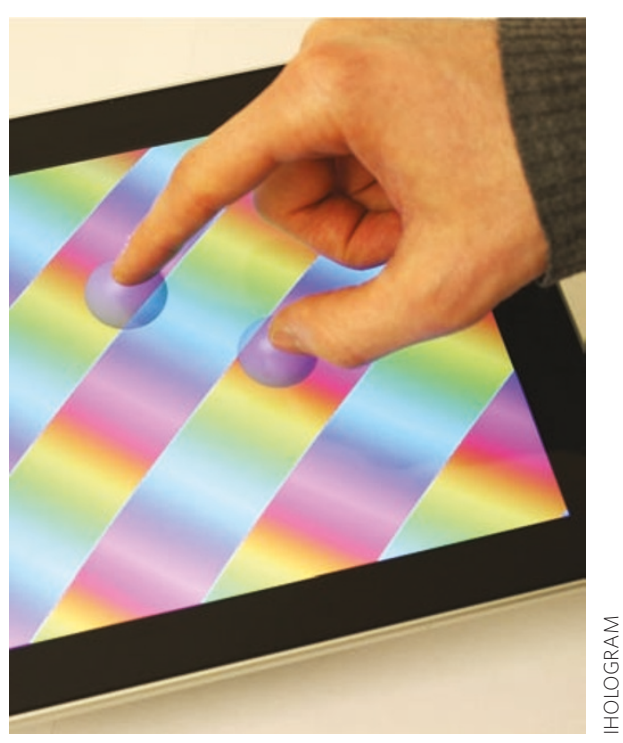

visualize the positions of several optical traps using the touch-screen of an iPad. Their next goal is to produce a more general toolkit that allows mobile devices to interact with any equipment controlled by National Instrument's LabVIEW software.

Scientists may soon be able to use tablet devices as interactive lab notebooks not only to collect, organize and display experimental results in real time, but also to provide an intuitive touch-screen interface for controlling their scientific apparatus. In the meantime, if you have come across any apps that you've found particularly useful as a researcher in optics, then please do write in and tell us about them.

\section{Business as usual}

\section{Despite recent events in Japan, Nature Photonics is still functioning.}

Many readers will be aware that the editorial and production headquarters of Nature Photonics are located in Tokyo, Japan, and have been ever since the launch of the journal in January 2007. Following the appalling earthquake and tsunami that struck the country on 11 March, we have received many queries regarding its effect on our operations and the well being of our staff. In response, we would like to reassure our authors, reviewers and readers that, despite some initial disruption, Nature Photonics is still functioning normally (thanks to additional support from the
NPG office in London) and that fortunately none of our staff in Japan were harmed. As we go to press, our Tokyo office is temporarily closed and staff are working from alternative locations until it reopens. In the meantime, please do not stop sending us your papers. 